\title{
Successful Nonoperative Management of Acute Spontaneous Idiopathic Spinal Epidural Hematoma Presenting as Cauda Equina Syndrome
}

\author{
Simranjeet Singh ${ }^{1}$ Amol Raheja ${ }^{1}$ Ashok K. Mahapatra \\ ${ }^{1}$ Department of Neurosurgery, All India Institute of Medical \\ Sciences, Ansari Nagar, New Delhi, India
}

Indian J Neurosurg 2021;10:258-259.
Address for correspondence Amol Raheja, Department of Neurosurgery, Neurosciences Centre, All India Institute of Medical Sciences, New Delhi 110029, India (e-mail: dramolraheja@gmail.com).
Spontaneous spinal epidural hematoma (SSEH) is a rare neurosurgical condition with an estimated incidence of 0.1 in $1,00,000$ population. ${ }^{1}$ It has been most commonly described in the older age group in the 4 th or 5 th decade of life and most commonly found in the dorsal or dorsolumbar region. ${ }^{2}$ It can present with a variety of clinical symptomatology, depending upon the site and extent of hematoma, usually in the form of acute cervical or back pain along with rapid onset of neurological deficits, often within first 12 hours of ictus and often requiring urgent neurosurgical intervention. However, its first manifestation as cauda equina syndrome is rare. Emergent surgical evacuation has remained the gold standard of treatment, ${ }^{3}$ and only a handful of cases reported successful recovery with conservative management, and that too primarily in patients with a diffuse SSEH associated with blood dyscrasias or coagulopathy. ${ }^{4}$ We describe a case of a 25-year-old healthy male, who presented with history of sudden onset severe back pain, followed by rapidly progressive paraplegia and cauda equina syndrome, four days after lifting heavy weight. He had to be catheterized for urinary retention. He presented to our clinic 1 month after the onset of symptoms, and by then, partial recovery of sensory, motor and autonomic deficits had already ensued. The coagulation profile was within normal parameters. His MRI performed immediately after the onset of symptoms demonstrated a T2-hyperintense lesion in posterior epidural space at L2-3 vertebral level, which was suggestive of a focal epidural hematoma with significant secondary canal compromise ( - Fig. 1 A,B). There was no evidence of associated flow-voids to suggest an associated vascular malformation. Due to delayed referral of patient and subacute stage of epidural hematoma with partial spontaneous recovery at the time of presentation, decision was taken to manage it conservatively with symptomatic pain management and limb physiotherapy. He recovered nearly completely over the next 1 month, and he was able to walk with minimal support and regained bladder function (American Spinal Injury Association [ASIA] D). His interval MRI demonstrated complete resolution of epidural hematoma ( $\mathbf{- F i g .} 2$ A,B).

The unique aspects of this reported case is the occurrence of idiopathic SSEH in a healthy young male with no underlying coagulopathy, vascular malformation, or direct trauma. The plausible explanation for the observed phenomenon could be the sudden increase in axial loading of spine, leading to increase in pressure within extradural venous plexus which, in turn, leads to the development of epidural hematoma. However, a delay of 4 days between axial loading of the spine and symptom onset is hard to explain, except for the fact that epidural hematoma started exerting mass effect only after it organized in a few days after the actual bleed. Second, cauda equina syndrome as the initial presentation for idiopathic SSEH is rarely reported in the current literature. Hence, SSEH should be kept as a differential diagnosis in a healthy male presenting with cauda equina syndrome, especially if associated with axial weight loading of the spine. Finally, the delayed presentation of patient to our clinic, subacute stage of the epidural hematoma at the time of presentation, and partial spontaneous recovery (from ASIA A to ASIA C) led us to proceed with conservative (nonoperative) management of SSEH, which led to successful recovery of neurological deficits and complete radiological resolution of hematoma. This raises an important question regarding the natural history of SSEH, optimal timing of surgical intervention published online

September 3, 2021
DOI https://doi.org/

10.1055/s-0041-1735428 ISSN 2277-954X
(C) 2021. Neurological Surgeons' Society of India.

This is an open access article published by Thieme under the terms of the Creative Commons Attribution-NonDerivative-NonCommercial-License, permitting copying and reproduction so long as the original work is given appropriate credit. Contents may not be used for commercial purposes, or adapted, remixed, transformed or built upon. (https://creativecommons.org/licenses/by-nc-nd/4.0/).

Thieme Medical and Scientific Publishers Pvt. Ltd. A-12, 2nd Floor, Sector 2, Noida-201301 UP, India 
(if at all required), and appropriate mode of therapeutic management (conservative versus operative). Due to the

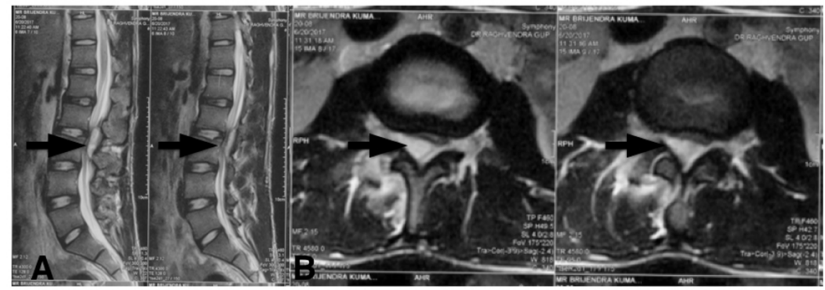

Fig. 1 Sagittal (A) and axial (B) T2-weighted MR imaging of spine, demonstrating hyperintense posterior epidural lesion (marked with arrow) suggestive of epidural hematoma with secondary canal compromise.

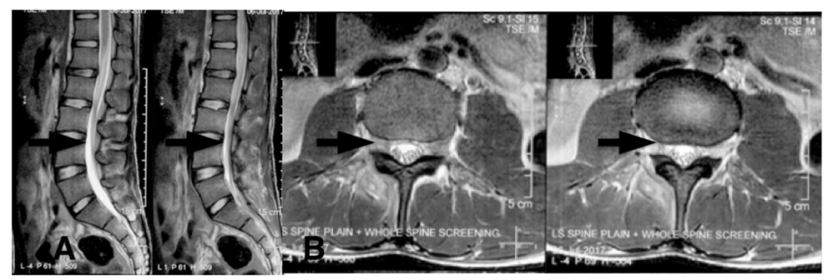

Fig. 2 Sagittal (A) and axial (B) T2-weighted interval MR imaging of spine (1 month follow-up), demonstrating complete resolution of epidural hematoma (marked with arrow) with no evidence of residual canal compromise. rarity of this disease, many of these questions still remain unanswered. Despite the controversy in literature, the majority believe that patient's neurological status, the timing of presentation, and the pattern of spontaneous clinical recovery are the primary determinants of the mode of therapeutic management (conservative or operative). Hence, a prospectively maintained multinational registry of SSEH could help better understand its etiopathogenesis, natural history, and optimal management techniques.

\section{Conflict of Interest}

None declared.

\section{References}

1 Holtås S, Heiling M, Lönntoft M. Spontaneous spinal epidural hematoma: findings at MR imaging and clinical correlation. Radiology 1996;199(2):409-413

2 Liu Z, Jiao Q, Xu J, Wang X, Li S, You C. Spontaneous spinal epidural hematoma: analysis of 23 cases. Surg Neurol 2008;69(3):253-260, discussion 260

3 Kim T, Lee CH, Hyun SJ, Yoon SH, Kim KJ, Kim HJ. Clinical outcomes of spontaneous spinal epidural hematoma: a comparative study between conservative and surgical treatment. J Korean Neurosurg Soc 2012;52(6):523-527

4 DuYll J, Sparrow OC, Millar J, Barker CS. Can spontaneous spinal epidural haematoma be managed safely without operation? A report of four cases. Korean J Spine 2011;8:272-282 